TRANSACTIONS OF THE

AMERICAN MATHEMATICAL SOCIETY

Volume 192, 1974

\title{
LOWER SEMICONTINUITY OF INTEGRAL FUNCTIONALS
}

\author{
BY \\ LEONARD D. BERKOVITZ(1)
}

\begin{abstract}
It is shown that the integral functional $I(y, z)=\int_{G} f(t, y(t), z(t)) d \mu$ is lower semicontinuous on its domain with respect to the joint strong convergence of $y_{k} \rightarrow y$ in $L_{p}(G)$ and the weak convergence of $z_{k} \rightarrow 2$ in $L_{q}(G)$, where $1 \leq p \leq \infty$ and $1 \leq q \leq \infty$, under the following conditions. The function $f:(t, x, w) \rightarrow f(t, x, w)$ is measurable in $t$ for fixed $(x, w)$, is continuous in $(x, w)$ for a.e. $t$, and is convex in $w$ for fixed $(t, x)$.
\end{abstract}

1. Introduction. To show that a functional attains a minimum on a given subset $\delta$ of its domain of definition $\mathscr{D}$ the following procedure is commonly used. One first shows that for an appropriate topology on $\mathscr{D}$ the set $\delta$ is compact and then one shows that the functional is lower semicontinuous on $\delta$ with respect to the topology in question. In this paper we shall consider the lower semicontinuity of certain integral functionals that arise in various minimization problems.

In [3] F. Browder studied the weak sequential lower semicontinuity of the functional

$$
J(\phi)=\int_{G} f(t,(M \phi)(t),(L \phi)(t)) d \mu,
$$

where $G$ is a bounded open set in $R^{n}, \mu$ is a finite regular Borel measure on $G, \phi$ is an element of a Banach space $\mathscr{F}, L$ is a bounded linear mapping of $\mathscr{F}$ into $L_{p}(G)$ and $M$ is a compact linear mapping of $\mathscr{F}$ into $L_{q}(G)$. Browder obtained two principal results [3, Theorems 4 and 5]. The first result involved certain Hölder type conditions on $f$ and has applications to elliptic boundary value problems. The second result involved certain growth conditions on $f$ and simplified some proofs and strengthened some theorems of Fichera [4] as well as some classical results in the calculus of variations. For further bibliographic details and discussion of applications see [3] and [4].

B. T. Poljak [9] studied the semicontinuity of the functional

$$
I(y, z)=\int_{G} f(t, y(t), z(t)) d \mu
$$

with respect to convergence of sequences $\left(y_{k}, z_{k}\right) \rightarrow(y, z)$, where the convergence of $y_{k}$ is convergence in $L_{p}(G)$ and the convergence of $z_{k}$ is weak

Received by the editors February 27, 1973.

AMS (MOS) subject classifications (1970). Primary 49A15, 49A20, 49A30.

Key words and phrases. Lower semicontinuity, optimal control of distributed parameter systems, existence theorems in variational problems.

(1) This research was supported by National Science Foundation Grant GP-33551X.

Copyright $\odot$ 1974, American Mathematical Society 
convergence in $L_{q}(G), 1 \leq p \leq \infty, 1 \leq q \leq \infty$. Here $p$ and $q$ need not be conjugate indices. Poljak's result [9, Theorem 12] was an improvement over Browder's Theorem 4 in that weaker continuity properties on $f$ and less restrictive Hölder type conditions were assumed.

In this paper we shall also study the semicontinuity of (1.2) with respect to the convergence studied in [9]. Our result in Theorem 1 below will be appreciably stronger than that of Poljak (and hence stronger than Theorem 4 of [3]) in that no Hölder type condition on $f$ will be assumed. Theorem 1 below will also include Theorem 5 of [3] and a classical theorem of Morrey [7, Theorem 4.1]. It can also be used to generalize some of the existence theorems of Lions [6] for problems of optimal control. Some of the principal ideas of the proof were used by us in investigations concerned with the existence of optimal controls in nonlinear control problems [1], [2].

A result similar to our Corollary 1 , with $p=q=2$, was given by V. I. Plotnikov [8, Lemma A]. Our proof is completely different from Plotnikov's and is much simpler than his.

2. Notation and definitions. We shall use single letters to denote vectors, we shall use subscripts to distinguish vectors, and we shall use superscripts to denote components of vectors. The letter $t$ will denote a vector $\left(t^{1}, \ldots, t^{\nu}\right)$ in real euclidean space $R^{\nu}, \nu \geq 1$, the letter $x$ will denote a vector $\left(x^{1}, \ldots, x^{n}\right)$ in $R^{n}$, and the letter $w$ a vector in $R^{m}, m^{\prime} \geq 1$. The euclidean norm of a vector will be denoted by $|x|$. The inner product of two vectors $x_{1}$ and $x_{2}$ will be written as $\left\langle x_{1}, x_{2}\right\rangle$. Thus $|x|=(\langle x, x\rangle)^{1 / 2}$.

Let $f:(t, x, w) \rightarrow f(t, x, w)$ be a real valued function defined in $R^{\prime} \times R^{n} \times R^{m}$. Let $G$ be a bounded open region on the $t$-space $R^{\nu}$ and let $d \mu$ be a finite regular Borel measure on $G$. Let $\mathscr{Y}$ denote the Banach space of functions $y=\left(y^{1}, \ldots\right.$, $y^{n}$ ) defined on $G$ with range in $R^{n}$ whose components $y^{i}$ are in $L_{p_{i}}(G)$, with respect to $d \mu$, where $1 \leq p_{i} \leq \infty$. The norm of an element $y=\left(y^{1}, \ldots, y^{n}\right)$ in O is given by

$$
\|y\|=\left(\sum_{i=1}^{n}\left\|y^{i}\right\|_{p_{i}}^{2}\right)^{1 / 2}
$$

where $\left\|y^{i}\right\|_{p_{i}}$ denotes the $L_{p_{i}}(G)$ norm of $y^{i}$. Let $\mathscr{Z}$ denote the Banach space of functions $z=\left(z^{1}, \ldots, z^{m}\right)$ defined on $G$ with range in $R^{m}$ whose components are in $L_{q_{i}}(G)$ with respect to $d \mu, 1 \leq q_{i} \leq \infty$, and where $\|z\|$ is defined in a manner analogous to $\|y\|$.

A sequence $\left\{\left(y_{k}, z_{k}\right)\right\}$ in $\mathcal{Y} \times \mathscr{Z}$ is said to converge to an element $(y, z)$ in $\mathcal{Y} \times \mathscr{Z}$ strongly in $y$ and weakly in $z$ if $\lim \left(y_{k}, z_{k}\right)=(y, z)$ in the product topology of Y $\times \mathscr{Z}$ determined by the strong topology of $\mathscr{Y}$ and the weak topology of $\mathscr{E}$

Let $\mathscr{D}$ denote the set of elements $(y, z)$ in $\mathscr{Y} \times \mathscr{Z}$ for which the integral in (1.2) exists and is finite. Then $\mathscr{D}$ is the domain of the functional $I$. We henceforth assume that $\mathscr{D}$ is not empty. 


\section{Statement and discussion of theorem.}

Theorem 1. Let $f$ be continuous in $(x, w)$ for a.e. $t$ in $G$ and let $f$ be measurable on $G$ for each $(x, w)$ in $R^{n+m}$. For each $(t, x)$ let $f$ be a convex function of $w$. Let $\psi$ be a function in $L_{1}(G)$ such that for all $(x, w)$ in $R^{n+m}$ and a.e. $t$ in $G$

$$
f(t, x, w) \geq \psi(t) .
$$

If $\left\{\left(y_{k}, z_{k}\right)\right\}$ is a sequence in $\mathscr{D}$ converging to an element $(y, z)$ in $\mathcal{Y} \times \mathscr{Z}$ strongly in $y$ and weakly in $z$ and if $\lim \inf I\left(y_{k}, z_{k}\right)<\infty$, then $(y, z)$ is in $\mathscr{D}$ and

$$
\lim \inf I\left(y_{k}, z_{k}\right) \geq I(y, z) \text {. }
$$

Note that “ $(y, z)$ is in $\mathscr{D}$ " is a conclusion of the theorem and not an assumption.

If we assume $(y, z)$ to be in $\mathscr{D}$ and if $\lim \inf I\left(y_{k}, z_{k}\right)=+\infty$, then the relation (3.2) automatically holds. Thus, we have the following corollary.

Corollary. The functional $I$ is sequentially lower semicontinuous on 10 with respect to strong convergence in $y$ and weak convergence in $z$.

Remark 1. The condition (3.1) is used in the proof to show that lim inf $I\left(y_{k}, z_{k}\right)$ $>-\infty$ and to justify the use of Fatou's lemma at a certain point. Upon reading the proof it will be clear that the condition (3.1) can be replaced by certain weaker conditions as in Poljak's paper. For example if all $p_{i}<\infty$ and all $q_{i}<\infty$ we can replace (3.1) by the condition

$$
f(t, x, w) \geq \psi(t)-\beta\left(\sum_{i=1}^{n}\left|x^{i}\right|^{p_{i}}+\sum_{i=1}^{m}\left|w^{i}\right|^{q_{i}}\right),
$$

where $\beta>0$. If all $p_{i}=\infty$ and all $q_{i}<\infty$, then we may replace (3.1) by the following condition. For each $M>0$, there exist a function $\psi_{M}$ in $L_{1}(G)$ and a constant $\beta_{M}>0$ such that for all $x$ in $R^{n}$ with $|x| \leq M$, all $w$ in $R^{m}$ and a.e. $t$ in $G$

$$
f(t, x, w) \geq \psi_{M}(t)-\beta_{M} \sum_{i=1}^{m}\left|w^{i}\right|^{q_{i}} .
$$

A similar condition can be imposed if all $q_{i}=\infty$ and all $p_{i}<\infty$. Finally, if all $p_{i}=\infty$ and all $q_{i}=\infty$ then we need merely require that for each $M>0$ there is a function $\psi_{M}$ in $L_{1}(G)$ such that (3.1) holds with $\psi$ replaced by $\psi_{M}$ for all $|x| \leq M,|w| \leq M$, and a.e. $t$ in $G$.

Remark 2. In [7, Theorem 4.1] Morrey showed that for $f$ continuous, $f$ convex in $w$ and appropriate assumptions on the boundary of $G$, the functional $\int_{G} f(t, \phi, \nabla \phi) d t$ is sequentially lower semicontinuous on its domain $\mathscr{D}$ with respect to weak convergence of sequences $\left\{\phi_{k}\right\}$ in $H_{1}^{1}(G)$. If $\phi_{k} \rightarrow \phi$ weakly in $H_{1}^{1}(G)$ then $\nabla \phi_{k} \rightarrow \nabla \phi$ weakly in $L_{1}(G)$. Also, for the region $G$ studied by Morrey, $\phi_{k} \rightarrow \phi$ weakly in $H_{1}^{1}(G)$ implies $\phi_{k} \rightarrow \phi$ in $L_{1}(G)$. Thus, Morrey's result follows from our corollary. 
Remark 3. In the introduction we discussed the relationship of our results to those of Poljak [9] and hence also to Theorem 4 of [3]. In Theorem 5 of [3] the integrand $f$ in (1.1) is assumed to be continuous in $(t, x, w)$ and convex in $w$, but is not assumed to satisfy a Hölder condition. Instead, it is assumed that there is a function $f_{0}$ that is convex in $w$ such that $f_{0}(w) /|w| \rightarrow \infty$ as $|w| \rightarrow \infty$ and such that $f(t, x, w) \geq f_{0}(w)$. It is not hard to see that this assumption implies that $f$ is bounded from below by an integrable function as in (3.1). Since in (1.1) the mapping $L$ is bounded and the mapping $M$ is compact it follows that if $\phi_{k} \rightarrow \phi$ weakly in $\mathscr{F}$ then $L \phi_{k} \rightarrow L \phi$ weakly in $L_{q}(G)$ and $M \phi_{k} \rightarrow M \phi$ strongly in $L_{p}(G)$. Hence if for $\phi$ in $\mathscr{F}$ we set $z=L \phi$ and $y=M \phi$, we get Theorem 5 of [3].

Remark 4. In some problems of optimal control it is required to minimize the functional (1.2) subject to contol constraints involving $z$ (the control) and the socalled "state equation" constraints, which involve $z, y$ (the state) and differential operators applied to $y$. In problems in which the state equations are linear in $w$ (the control variable) and $x$ (the state variable) and the differential operators are linear, a semicontinuity theorem for the functional is quite often used as one of the cornerstones of the proof.

In his book, Lions [6] usually considers quadratic functionals in which $f$ is essentially of the form

$$
f(t, x, w)=\langle x, Q(t) x\rangle+\langle b, x\rangle+\langle w, R(t) w\rangle
$$

and uses well-known results about the lower semicontinuity of such functionals. Theorem 1 enables one to extend many of the results of Lions to more general functionals (1.2) in which $f$ satisfies the hypotheses of Theorem 1. Other examples of control problems in which one requires the semicontinuity of the functional (1.2) can be found in [8] and [9].

4. Proof of Theorem 1. The following lemma is crucial to the proof of Theorem 1.

Lemma 1. Let $g:(t, x) \rightarrow g(t, x)$ be a mapping from $G \times R^{r} \rightarrow R^{1}$ that is continuous in $x$ for a.e. $t$ in $G$ and measurable in $t$ for fixed $x$ in $R^{r}$. Let $\left\{v_{k}\right\}$ and $\left\{u_{k}\right\}$ be sequences in $\mathscr{B}=\prod_{i=1}^{r} L_{s_{i}}(G)$ such that $\left\|u_{k}\right\| \leq R$ and $\left\|v_{k}\right\| \leq R$ for some $R>0$ and such that $\left(u_{k}-v_{k}\right) \rightarrow 0$ in measure on $G$. Then

$$
g\left(t, u_{k}(t)\right)-g\left(t, v_{k}(t)\right) \rightarrow 0
$$

in measure on $G$.

A proof of this lemma can be found in [5, p. 355]. In [5] it is assumed that all of the $s_{i}$ are equal, say $s_{i}=s$. To obtain the statement used here take $s=\min s_{i}$ and recall that $G$ has finite measure.

In the course of the proof of Theorem 1 we shall select subsequences of various sequences. Unless stated otherwise we shall relabel the subsequence with the labeling of the original sequence. 
Let

$$
\gamma=\lim \inf I\left(y_{k}, z_{k}\right) \text {. }
$$

By virtue of (3.1), $\gamma>-\infty$. Since by hypothesis $\gamma<\infty$, we have that $\gamma$ is finite. We first select a subsequence $\left\{\left(y_{k}, z_{k}\right)\right\}$ such that

$$
\lim I\left(y_{k}, z_{k}\right)=\gamma .
$$

From the definition of $\mathcal{Y}$ in $\$ 2$ it follows that the sequences of components $\left\{y_{k}^{l}\right\}$ converge in $L_{p_{i}}(G)$ to $y^{i}$. Hence $y_{k} \rightarrow y$ in measure on $G$.

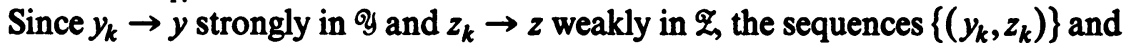

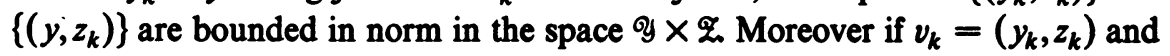
$u_{k}=\left(y, z_{k}\right)$, then $v_{k}-u_{k} \rightarrow 0$ in measure. Let

$$
h_{k}(t)=f\left(t, y_{k}(t), z_{k}(t)\right)-f\left(t, y(t), z_{k}(t)\right)
$$

then by Lemma $1, h_{k} \rightarrow 0$ in measure on $G$.

Since $h_{k} \rightarrow 0$ in measure, there exists a subsequence $\left\{\left(y_{k}, z_{k}\right)\right\}$ such that for a.e. $t$ in $G$

$$
h_{k}(t) \rightarrow 0 \text {. }
$$

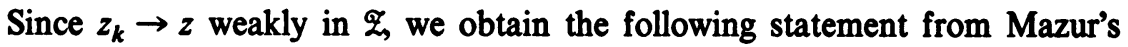
theorem. For each integer $j$ there exists an integer $n_{j}$, a set of integers $i=1, \ldots, k$, where $k=k(j)$ depends on $j$, and a set of numbers $\alpha_{y}, \ldots, \alpha_{k j}$ satisfying

$$
\alpha_{i j} \geq 0, \quad \sum_{i=1}^{k} \alpha_{i j}=1,
$$

such that $n_{j+1}>n_{j}+k(j)$ and

$$
\left\|z-\sum_{i=1}^{k} \alpha_{i j} z_{n_{j}+i}\right\|<\frac{1}{j} .
$$

Let

$$
\psi_{j}=\sum_{i=1}^{k} \alpha_{i j} z_{n+i} .
$$

From (4.6) we have that $\psi_{j} \rightarrow z$ in $\mathscr{\mathcal { L }}$ Hence there is a subsequence $\left\{\psi_{j}\right\}$ such that

$$
\psi_{j}(t) \rightarrow z(t) \quad \text { a.e. }
$$

We now suppose that (4.7) is the subsequence for which (4.8) holds. Corresponding to (4.7) we define a sequence $\left\{\lambda_{j}\right\}$ as follows: 


$$
\lambda_{j}(t)=\sum_{i=1}^{k} \alpha_{i j} f\left(t, y_{n j+i}(t), z_{n j+i}(t)\right),
$$

where for each $j$ the numbers $\alpha_{i j}$, the indices $n_{j}+i$, and hence the functions $z_{n j+b}$, are as in (4.7). Define $\lambda(t)=\lim \inf \lambda_{j}(t)$. From (4.9), (4.5), and (3.1) we get that for each $j$ and a.e. $t$ in $G$

$$
\lambda_{j}(t) \geq \sum_{i=1}^{k} \alpha_{i j} \psi(t)=\psi(t)
$$

We may therefore apply Fatou's lemma and get that

$$
\begin{aligned}
\int_{G} \lambda(t) d \mu & \leq \lim \inf \int_{G} \lambda_{j}(t) d \mu \\
& =\liminf \sum_{i=1}^{k} \alpha_{i j} \int_{G} f\left(t, y_{n j+i}(t), z_{n j+i}(t)\right) d \mu \\
& =\lim \inf \sum_{i=1}^{k} \alpha_{i j} I\left(y_{n j+i}, z_{n j+i}\right) .
\end{aligned}
$$

We had originally selected a subsequence $\left\{\left(y_{k}, z_{k}\right)\right\}$ such that $I\left(y_{k}, z_{k}\right) \rightarrow \gamma$. Therefore it follows from (4.5) that

$$
\int_{G} \lambda(t) d \mu \leq \gamma
$$

From (4.10) we get that $\lambda(t) \geq \psi(t)$ a.e. Hence $\lambda$ is finite a.e.

Let $G_{1}$ denote the set of points at which $\lambda(t)$ is infinite. Let $G_{2}$ denote the set of points at which (4.4) fails and let $G_{3}$ denote the set of points at which (4.8) fails. Let $G_{4}$ denote the set of points in $G$ at which $f$ is not continuous in $(x, w)$. Let $G_{5}=\bigcup_{i=1}^{4} G_{i}$. The $\mu$-measure of $G_{5}$ is zero. Let $G^{\prime}=G-G_{4}$.

Let

$$
\omega_{j}(t)=\sum_{i=1}^{k} \alpha_{i j} f\left(t, y(t), z_{n j+i}(t)\right),
$$

where the numbers $\alpha_{i j}, i=1, \ldots, k$, the indices $n_{j}+i$ and the functions $z_{n j+l}$ are as in (4.7). From (4.9) and (4.3) we have

$$
\lambda_{j}(t)-\omega_{j}(t)=\sum_{i=1}^{k} \alpha_{i j} h_{n j+i}(t)
$$

Since $h_{k}(t) \rightarrow 0$ on $G^{\prime}$, it follows from (4.5) that

$$
\lambda_{j}(t)-\omega_{j}(t) \rightarrow 0 \text { on } G^{\prime}
$$

Let $t$ be an arbitrary point in $G^{\prime}$. There exists a subsequence, which may depend on $t$, such that $\lambda_{j}(t) \rightarrow \lambda(t)$. For this subsequence we have $\omega_{j}(t) \rightarrow \lambda(t)$ by virtue of (4.12). Since for fixed $(t, x), f$ is a convex function of $w$, we have 


$$
\omega_{j}(t)=\sum_{i=1}^{k} \alpha_{i j} f\left(t, y(t), z_{n j+i}(t)\right) \geq f\left(t, y(t), \psi_{j}(t)\right),
$$

where we have used (4.5) and (4.7). If we now let $j \rightarrow \infty$ and make use of (4.8), the continuity of $f$ with respect to $w$, and the fact that $\omega_{j}(t) \rightarrow \lambda(t)$ for the point $t$ and subsequence in question, we get

$$
\lambda(t) \geq f(t, y(t), z(t)) .
$$

Since $t$ was an arbitrary point of $G^{\prime}$, we have that (4.13) holds a.e. in $G$.

Under our hypothesis on $f$ the mapping $t \rightarrow f(t, y(t), z(t))$ is measurable. It then follows from (4.13) and (3.1) that $f$ is in $L_{1}(G)$ and that

$$
\int_{G} \lambda(t) d \mu \geq \int_{G} f(t, y(t), z(t)) d \mu>-\infty .
$$

Thus $(y, z)$ is in $\mathscr{D}$.

From (4.11) and the preceding inequality we get

$$
\gamma \geq \int_{G} f(t, y(t), z(t)) d \mu .
$$

The relation (3.2) now follows from the definition of $\gamma$ in (4.1) as $\lim$ inf $I\left(y_{k}, z_{k}\right)$, where $\left\{\left(y_{k}, z_{k}\right)\right\}$ is the original sequence.

\section{REFERENCES}

1. L. D. Berkovitz, Existence theorems in problems of optimal control, Studia Math. 44 (1972), 275-285.

2.—, Existence and lower closure theorems for abstract control problems, SIAM J. Control (to appear).

3. F. Browder, Remarks on the direct method of the calculus of variations, Arch. Rational Mech. Anal. 20 (1965), 251-258. MR 32 \#4576.

4. G. Fichera, Semicontinuity of multiple integrals in ordinary form, Arch. Rational Mech. Anal. 17 (1964), 339-352. MR 29 \#6354.

5. M. A. Krasnosel'skii, P. P. Zabreiko, E. I. Pustyl'nik and P. E. Sobolevskiī, Integral operators in spaces of summable functions, "Nauka", Moscow, 1966. (Russian) MR 34 \#6568.

6. J. L. Lions, Optimal control of systems governed by partial differential equations, Die Grundlehren der math. Wissenschaften, Band 170, Springer-Verlag, Berlin and New York, 1971. MR 42 \#6395.

7. C. B. Morrey, Jr., Multiple integral problems in the calculus of variations and related topics, Univ. California Publ. Math. 1 (1943), 1-130. MR 6, 180.

8. V. I. Plotnikov, Existence theorems on optimizing functions for optimal systems with distributed parameters, Izv. Akad. Nauk SSSR Ser. Mat. 34 (1970), 689-712 = Math. USSR Izv. 4 (1970), 697-719. MR 41 \# 7242.

9. B. T. Poljak, Semicontinuity of integral functionals and existence theorems on extremal problems, Mat. Sb. 78120 (1969), 65-84 = Math. USSR Sb. 7 (1969), 59-77. MR 39 \#6140.

Department of Mathematics, Purdue University, West LafayetTe, Indiana 47907 